\title{
Lernende Klassifizierungssysteme zur fallbasierten Auswertung von Allergietestdaten
}

\author{
J. Albert ${ }^{1}$, J. Geier ${ }^{2}$, M. Lehmann ${ }^{1}$, J. Schoof ${ }^{1}$ \\ ${ }^{1}$ Informatik II, Universität Würzburg; \\ ${ }^{2}$ Informationsverbund Dermatologischer Kliniken (IVDK) Göttingen
}

Das Problem des Aufspürens von signifikanten Zusammenhängen in den Falldaten zu Allergietests kann als eine Aufgabenstellung des sog. Data Mining behandelt werden. In einem interdisziplinären Projekt werden hierzu unterschiedliche Methoden des maschinellen Lernens eingesetzt und deren Leistungsfähigkeit verglichen.

Die Induktion von Regeln mit Hilfe eines bekannten Verfahrens von Clark und Niblett (CN2) wurde inzwischen durch „Learning Classifier Systems“ (LCS) erweitert. Diese sind in der Lage, aus einer Sammlung von Falldaten unter der Kontrolle von variablen Kostenmaßen kompakte Regel- listen zu generieren. Durch die Verwendung heuristischer Methoden, die der künstlichen Evolution zuzuordnen sind, wird es möglich, dabei auch solche Regeln zu finden, die bei anderen Verfahren aus systematischen Gründen unentdeckt bleiben. Weiterhin bietet der Rahmen der künstlichen Evolution eine Reihe von Möglichkeiten, Experten interaktiv an der Regelfindung zu beteiligen. Sie können u.a. dem LCS Vorschläge unterbreiten, die getestet, bewertet und ggf. übernommen werden. Auch andere Informationsquellen, wie RegelDatenbanken oder konventionelle statistikorientierte Programme, kön- nen zur Generierung von Vorschlägen integriert werden. Ein Prototyp eines solchen Systems konnte nun fertiggestellt werden. Erste Ergebnisse zeigen die Vergleichbarkeit mit Resultaten anderer Verfahren und Untersuchungen des IVDK bei großen Fallzahlen. Eine Feineinstellung der Steuerparameter des Prototyps ist zur Zeit in Arbeit.

Die oben genannten Vorteile des Systems sollen dem Nutzer künftig eine aktivere Rolle bei der Induktion von Regeln ermöglichen, was sich letztlich auf die Qualität der Resultate positiv auswirken dürfte.

\section{Untersuchungen zur Korrelation zwischen Basis-TEWL und Atopiescore nach Diepgen an einem Kollektiv Schweizer Metallarbeiterlehrlinge}

\author{
D. Iliev, U. Hinnen, P. Elsner \\ Dermatologische Klinik am Universitätsspital Zürich/Schweiz
}

Bei den Berufskrankheiten der Haut ist im Metallarbeiterberuf in der Schweiz innerhalb der letzten Jahre ein Zuwachs zu verzeichnen gewesen. Zur Einschätzung subklinischer ekzematöser Hautveränderungen hat sich der transepidermale Wasserverlust (TEWL) als wichtiger Parameter bewährt.

In dieser im Rahmen der Zürcher Prospektiven Metallarbeiter-EkzemStudie (PROMETES) durchgeführten Untersuchung sollte der Zusammenhang zwischen dem Atopiescore von 204 Metallarbeiterlehrlingen in den ersten Wochen ihrer Lehre und ihren TEWL-Werten an Handrücken und Unterarm-Innenseite beidseits untersucht werden.

Die Messungen erfolgten bei $18-22^{\circ} \mathrm{C}$ und wurden auf $30^{\circ} \mathrm{C}$ standardisiert. Zusätzlich wurde der Hautstatus an den Händen und Unterarmen erhoben.

Die Korrelationsanalyse nach Pearson zeigte keinen signifikanten Zusammenhang zwischen Atopiescores und TEWL-Werten an Handrücken und Unterarm-Innenseite beidseits auf. Nur zwei Probanden, beide mit einem Atopiescore von 3 nach Diepgen, wiesen einen geringgradigen Hautbefund mit einem Meding-Score (1) von 1 bzw. 4 auf.
Bei den TEWL-Werten dieser beiden Probanden konnte an keinem der drei Meßpunkte ein signifikanter Unterschied im Vergleich zur Vergleichspopulation ermittelt werden.

Diese Untersuchungen bestätigen Ergebnisse von Nöring und Mitarbeitern (2), wonach der Atopiescore und der TEWL zwei voneinander unabhängige Größen sind. Bei den beiden Lehrlingen mit Hautveränderungen der Hände konnte aufgrund des im Ausmaß sehr diskreten Befundes, der sich nicht über den Meßpunkten in der Mitte beider Handrücken befand, keine auffällige TEWL-Veränderung gemessen wer- 